\title{
BIBLIOGRAPHY
}

1. S. Ramanujan, Congruence properties of partitions, Math. Zeit. vol. 9 (1921) pp. 147-153. Reprinted in Collected papers of Srinivasa Ramanujan, Cambridge, 1927, pp. 232-238.

2. S. Ramanujan, On certain arithmetical functions, Trans. Cambridge Philos. Soc. vol. 22 (1916) pp. 159-184. Reprinted in Collected papers of Srinivasa Ramanujan, pp. $136-162$.

3. H. S. Zuckerman, Identities analogous to Ramanujan's identities involving the partition function, Duke Math. J. vol. 5 (1939) p. 89, equation (1.15).

UNIVERsity OF CALIFoRNia

\section{AUTOMORPHISMS OF FIELDS OF FORMAL POWER SERIES}

\section{O. F. G. SCHILLING}

We propose to discuss in this note on power series fields in one variable the special automorphisms which do not alter the fields of coefficients. It will be proved that the pseudo-ramification groups introduced by MacLane are universal ramification groups, in the sense that a special ramification group must always be a subgroup of a well determined pseudo-ramification group. Finally we interpret the automorphism group of the field as an automorphism group of an infinite Lie ring.

Let $\Omega$ be an arbitrary field of characteristic $\chi$. In the sequel we shall consider the field $F$ of all formal power series $a=\sum_{j>-\infty}^{\infty} \omega_{j} t^{i}$ where the $\omega_{j}$ are in $\Omega$ and $t$ is a transcendental element over $\Omega .{ }^{1}$ The field $F$ is complete with respect to the rank one valuation $V$ defined by $V a=m$ where $m$ is the smallest subscript $j$ for which $\omega_{j} \neq 0$. Let $\mathfrak{O}$ be the valuation ring of all holomorphic series and $\mathfrak{B}=(t)$ the principal prime ideal of $\mathfrak{D}$.

Suppose that $S$ is an automorphism of $F$. We show that $\mathfrak{D}^{S}$ is also a valuation ring of $F$. For the proof ${ }^{2}$ let $a, b$ be any two nonzero elements of $F$. We must show that at least one of the quotients $a / b, b / a$ lies in $D^{S}$. By assumption on $S$ there exist unique elements $c, d$ with $c^{S}=a, d^{S}=b$. Now observe that at least one of the quotients $c / d$ or $d / c$ lies in $\mathfrak{O}$ for $\mathfrak{O}$ is a valuation ring. Therefore at least one of the

Presented to the Society, August 14, 1944; received by the editors May 29, 1944.

${ }^{1}$ For the basic properties of valuations see $[1,4,5,10]$. Numbers in brackets refer to the bibliography at the end of the paper.

2 See $[4$, p. 165]. 
elements $(c / d)^{S}=a / b$ or $(d / c)^{S}=b / a$ lies in $\mathcal{D}^{S}$. Furthermore we observe that the ideal theory of $D$ is carried over isomorphically to $D^{S}$. Thus $\mathfrak{D}^{S}$ defines a rank one valuation $V^{S}$ on $F$.

Definition $1 .^{3}$ An automorphism $S$ of $F$ is called analytic if $V a<V b$ is equivalent to $V^{s} a^{s} \leqq V^{s} b^{s}$ for each pair of elements $a, b$ in $F$.

LEMMA 1. Each automorphism of $F$ is analytic.

Proof. We shall prove that $F$ is complete with respect to the valuation $V^{S}$. Let $a_{n} \equiv a_{n-1}\left(\bmod \left(\mathfrak{P}^{S}\right)^{n}\right)$ be an infinite consistent system of congruences with respect to $V^{S}$. There exist uniquely determined elements $c_{n}$ with $c_{n}^{S}=a_{n}$ and we have $c_{n} \equiv c_{n-1}\left(\bmod \mathfrak{P}^{n}\right)$. Hence there exists an element $x$ in $F$ with $x \equiv c_{n}\left(\bmod \mathfrak{P}^{n}\right)$, for $F$ is complete with respect to $V$. Applying $S$ to $x$ we have $x^{S} \equiv c_{n}^{S} \equiv a_{n}\left(\bmod \left(\mathfrak{P}^{S}\right)^{n}\right)$. Hence $F$ is complete with respect to $V^{S}$. Consequently $F$ would be multiply complete if $V$ and $V^{S}$ were distinct valuations. Thus it would follow that $F$ is algebraically closed, in contradiction to the construction of $F$. Hence $V$ and $V^{s}$ are equivalent valuations, that is, $S$ is analytic.

Suppose that $s$ is an automorphism of $\Omega$. If we observe the rules for the addition and multiplication of elements in $F$, the correspondence $\sum_{j>-\infty}^{\infty} \omega_{j} t^{j} \rightarrow \sum_{j>-\infty}^{\infty} \omega_{j}^{s} t^{j}=\left(\sum_{j>-\infty}^{\infty} \omega_{j} t^{j}\right)^{s}$ defines an automorphism of $F$. These automorphisms of $F$ determine a subgroup of the automorphism group $A$ of $F$ which is isomorphic with the automorphism group of $\Omega$. A simple computation shows that this subgroup is not normal if and only if it is not the trivial group consisting of the identity. Now let $T$ be an arbitrary automorphism of $F$. The element $T$ either induces an automorphism on $\Omega \subset F$ or it maps $\Omega$ into an isomorphic subfield $\Omega^{T} C F$. We shall consider only those automorphisms of $F$ for which all the elements of $\Omega$ are invariant. These automorphisms form a subgroup $G$ of $A$. This group $G$ corresponds to the inertial group considered in the theory of normal algebraic extensions of fields with valuations.

Lemma 2. A field $F$ which is complete with respect to a rank one valuation has no immediate extensions. ${ }^{5}$

Proof. Suppose that $K$ is an immediate extension of $F$. Let $A$ be an element of $K$. We shall show that $A$ must lie in $F$. By assumption the value of $A$ is the value of an element $a$ in $F$. Thus $A / a$ is a unit

${ }^{8}$ See $[5$, footnote on p. $373 ; 12]$.

${ }^{4}$ See [12].

See $[4$, p. 191]. 
and therefore $A / a \equiv \omega_{0}\left(\bmod \mathfrak{P}_{K}\right)$, where $\mathfrak{P}_{K}$ is the prime ideal of $K$ and $\omega_{0}$ is in $\Omega$. Next there is at least one element $a_{1}$ in $F$ with $A / a-\omega_{0} \equiv a_{1}\left(\bmod \mathfrak{P}_{K}^{n_{1}}\right)$, where $n_{1}=V\left(A / a-\omega_{0}\right)$. By complete induction there exists a sequence of elements $a \omega_{0}, a_{0}+a a_{1} \omega_{1}, \cdots, a \omega_{0}$ $+\cdots+a a_{1} \cdots a_{\nu} \omega_{\nu}, \cdots$ such that $A \equiv a \omega_{0}+\cdots+a a_{1} \cdots a_{\nu} \omega_{\nu}$ $\left(\bmod \mathfrak{P}_{K}^{m}\right)$ with $m=n_{1}+\cdots+n_{\nu} \rightarrow \infty$ for $\nu \rightarrow \infty$. This sequence has a limit $b$ in $F$ and $A-b$ has value $\infty$ in $K$. Hence $A=b$ in $F .^{6}$

Suppose that $S \neq 1$ is an automorphism of $F$ with $\omega=\omega^{S}$ for all $\omega$ in $\Omega$. Then $t^{S} \neq t$, for $t=t^{S}$ implies $a=a^{S}$ for every $a$ in $F$. Hence each $S \neq 1$ determines by $t^{S}=t u(S)$ an element $u(S)$ of the unit group $U$ of $F$.

THEOREM 1. The groups $G$ and $U$ are in one-to-one correspondence as sets.

Proof. As seen above each automorphism $S$ determines relative to the prime element $t$ a unit $u(S)$. For the converse we shall show that the mapping $t \rightarrow t^{*}=t u$ determines, for given $u$ in $U$, an automorphism of $F$. We associate to an arbitrary element $a=\sum_{j>-\infty}^{\infty} \omega_{j} t^{i}$ the quantity $a^{*}=\sum_{j>-\infty}^{\infty} \omega_{j}\left(t^{*}\right)^{i}$. The elements $a^{*}$ form by definition a subfield $F^{*}$ of $F$ which is isomorphic to $F$. By construction the valuation $V$ of $F$ induces a valuation $V^{*}$ on the complete field $F^{*}$ with $V^{*} t^{*}=V t^{*}=V t$ so that $F$ and $F^{*}$ have the same residue class field. Therefore $F$ is an immediate extension of $F^{*}$ and hence $F=F^{*}$ by Lemma 2 . Consequently the mapping $a \rightarrow a^{*}$ is an automorphism of $F$. We remark that the identity elements of $G$ and $U$ correspond to each other.

We next give a definition and a set of formulas which can be used to compute explicitly the coefficients of $a^{*}$ relative to the prime element $t$. We define the derivative $D_{t} a$ of $a=\sum \omega_{j} t^{t}$ as $\sum j \omega_{j} t^{i-1}$ and $D_{i}^{i}=(1 / i !)(d / d t)^{i}$. Then ${ }^{7}$

$$
\begin{aligned}
D_{t}^{i}(a+b) & =D_{t}^{i} a+D_{t}^{i} b, D_{t}^{i} t^{n}=C_{n, i} t^{n-i}, \quad i \geqq 0, \\
D_{t}^{i}(\omega a) & =\omega D_{t}^{i} a, \omega \text { in } \Omega, \\
D_{t}^{i}(a b) & =\sum_{i} D_{t}^{i_{1}} a D_{t}^{i_{2}} b ; \quad i_{1} \geqq 0, i_{2} \geqq 0, i_{1}+i_{2}=i .
\end{aligned}
$$

As in the calculus the inversion formula of Lagrange holds, $t=\sum_{j=1}^{\infty} \lambda_{j}\left(t^{*}\right)^{j}$ with $\lambda_{j}=\left[D_{t}^{j}\left\{D_{t} t^{*} \cdot\left(t / t^{*}\right)^{j}\right\}\right]_{t=0}, \lambda_{1} \neq 0$. Hence $t$ lies in $F^{*}$ and thus $F^{*}=F$. Using the Taylor developments of the elements $a^{*}=\sum \omega_{j}\left(t^{*}\right)^{i}=\sum D_{t}^{j}\left\{a^{*}\right\}_{t=0} t^{i}$ it follows that each unit $u$ determines by $t \rightarrow t^{*}=t u$ an automorphism of $F$. Letting $b^{*}=\sum \rho_{j}\left(t^{*}\right)^{j}$ $=\sum D_{t}^{\prime}\left(b^{*}\right)_{t=0} t^{i}$ we have, by the rules on the derivatives $D_{t}^{\prime}$,

- For another proof see [5, pp. 379-380].

' See [2]. 


$$
\begin{aligned}
(a+b)^{*} & =\sum\left\{D_{t}^{j}\left(a^{*}+b^{*}\right)\right\}_{t-0} t^{j} \\
& =\sum\left\{D_{t}^{j} a^{*}\right\}_{t=0} t^{j}+\sum\left\{D_{t}^{j} b^{*}\right\}_{t=0} t^{j}=a^{*}+b^{*}, \\
(a b)^{*} & =\sum\left\{D_{t}^{j}\left(a^{*} b^{*}\right)\right\}_{t=0} t^{j} \\
& =\sum \sum\left\{D_{t}^{j_{1}} a^{*}\right\}_{t=0}\left\{D_{t}^{j_{2}} b^{*}\right\}_{t=0} t^{j_{1}+i_{2}}=a^{*} b^{*},
\end{aligned}
$$

where $j_{1} \geqq 0, j_{2} \geqq 0, j_{1}+j_{2}=j$, and similarly $(a \omega)^{*}=a^{*} \omega$.

Now let $S, T$ be two automorphisms of $G$. On applying $S T$ to the prime element $t$ we find $t^{S T}=\left(t^{T}\right)^{S}=(t u(T))^{S}=t^{S} u(T)^{S}=t u(S) u(T)^{S}$ $=t u(S T)$ where the $u$ 's are the units corresponding to the automorphisms. Thus the mapping $S \rightarrow u(S)$ gives rise to a crossed representation $^{8}$ of the automorphism group $G$ in the unit group $U$, for $u(S T)$ $=u(S) u(T)^{S}$. The latter relation may be viewed as a new multiplication $\times$ between the elements of $U$. We define $u_{1} \times u_{2}$ to be $u_{1} u_{2}^{W}$ where $W=S\left(u_{1}\right)$ is the automorphism which corresponds to $u_{1}$ by virtue of Theorem 1. The associativity of the group $G$ implies that $U$ is a group with respect to the operation $X$.

We now proceed to a different interpretation of this operation. Let $\Omega_{x}$ be the set of all formal power series $\sum_{i=0}^{\infty} \omega_{i} x^{i}$, where $x$ is an indeterminate over $\Omega$.

Definition 2. If $A=\sum \alpha_{i} x^{i}$ and $B=\beta_{i} x^{i}$ then define $A \otimes B$ as $\sum_{i} \beta_{i}\left(\sum_{j} \alpha_{j} x^{j}\right)^{i}$ if and only if $\alpha_{0}=0$.

From the definition of $\Omega_{x}$ we observe that $\Omega_{x}$ may be considered as an infinite vector space over $\Omega$ with restricted multiplication. ${ }^{\circ}$ The element $x$ plays the role of a left and right unit on the subset $U$ of all elements without constant terms. The set $U$ is a group, as may be verified by using Taylor expansions with respect to $x$. Obviously the product of elements corresponds to the operation of taking the function of a function. It is now easy to set up a relation between $U$ with $X$ as operation and $U$. If $u=\sum_{j=0}^{\infty} \omega_{j} t^{j}, \omega_{0} \neq 0$, is given then we take for the corresponding element $u=\sum_{j=0}^{\infty} \omega_{j} x^{i+1}$. The product $u_{1} \times u_{2}$ of two elements $u_{1}, u_{2}$ in $U$ is then determined as $\left\{\left(u_{1} \otimes u_{2}\right) x^{-1}\right\}_{x=t}$ where the factor $x^{-1}$ is to indicate a scalar division of $u_{1} \otimes u_{2}$ by $x$. In the sequel it will be useful to use the representation of the elements $S$ in $G$ by means of the elements in $U ; S \rightleftarrows u(S) \rightleftarrows u(S)$. We shall use the symbol $S$ ambiguously for the automorphism $S$ and the representation $u(S)$.

Suppose now that $\mu_{1}, \mu_{2}$ are two nonzero elements of $\Omega$. These elements determine, by $t \rightarrow t \mu_{i}$, automorphisms $S\left(\mu_{i}\right)$ in $G, i=1,2$. The

${ }^{8}$ See $[7$, p. 313].

See $[8,9]$. 
multiplication rule of $U$ shows $S\left(\mu_{1}\right) S\left(\mu_{2}\right)=\left(x \mu_{1} \otimes x \mu_{2}\right)=S\left(\mu_{1} \mu_{2}\right)$ $=S\left(\mu_{2} \mu_{1}\right)=\left(x \mu_{2} \otimes x \mu_{1}\right)=S\left(\mu_{2}\right) S\left(\mu_{1}\right)$. Hence the multiplicative group $\Omega^{*}$ of $\Omega$ has an isomorphic image in $G$.

The group $G$ contains an infinite series of subsets $G_{i}$ defined by the elements $S_{i}=x+\sum_{\nu=i+1}^{\infty} \omega_{\nu} x^{\nu}$.

THEOREM 2. The sets $G_{i}$ are invariant subgroups of $G$, their intersection $\cap_{i} G_{i}$ is the identity and each factor group $G_{i} / G_{i+1}$ is isomorphic to the additive group $\Omega$. The factor group $G / G_{1}$ is isomorphic to the multiplicative group $\Omega^{*}$.

Proof. Let $S=x+\omega_{1} x^{i}+\cdots, T=x+\omega_{2} x^{i}+\cdots$ be two elements of $G_{i}$. Then, by definition of the product in $U$,

$$
\begin{aligned}
S \otimes T & =x+\left(\omega_{1}+\omega_{2}\right) x^{i}+\cdots, \\
T \otimes S & =x+\left(\omega_{2}+\omega_{1}\right) x^{i}+\cdots, \\
S^{-1} & =x+\left(-\omega_{1}\right) x^{i}+\cdots .
\end{aligned}
$$

Thus $G_{i}$ is a group. To show that $G_{i}$ is an invariant subgroup of $G$ let $W=\alpha_{0} x+\alpha_{1} x^{2}+\cdots$ be an arbitrary element of $G$. The inversion formula for power series implies $W^{-1}=\alpha_{0}^{-1} x+\left(-\alpha_{1} / \alpha_{0}^{3}\right) x^{2}+\cdots$. Consequently, by direct computation, $W \otimes S \otimes W^{-1}=x+\alpha_{0}^{i-1} \omega_{1} x^{i}$ $+\ldots$ lies also in $G_{i}$. Definition of the $G_{i}$ implies $G \supset G_{1} \supset \ldots$ and $\bigcap_{i} G_{i}=x$, the identity of $G$. The relations $\left(^{*}\right)$ imply that $G_{i} / G_{i+1}$ is isomorphic to the additive group $\Omega$. Now let $S\left(\alpha_{0}\right)$ be determined by the element $W=\alpha_{0} x+\alpha_{1} x^{2}+\cdots$. Then $S\left(\alpha_{0}\right)^{-1} \otimes W=\alpha_{0}\left(\alpha_{0}^{-1}\right) x$ $+\alpha_{1}\left(\alpha_{0}^{-1}\right) x^{2}+\cdots=x+\left(\alpha_{1} / \alpha_{0}\right) x^{2}+\cdots$ lies in $G_{1}$. Hence

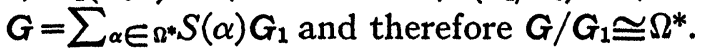

COROLlaRY 1. The group $G$ is a group extension of $G_{1}$ by $\Omega^{*}$ with factor set unity.

P.Roof. As seen before the elements $S(\mu), \mu$ in $\Omega^{*}$, form a subgroup of $G$. The elements $S(\mu)=\mu x$ induce automorphisms on the group $G_{1}=\left\{x+\omega_{1} x^{2}+\cdots+\omega_{n} x^{n+1}+\cdots\right\}$. The associated combinations are determined by

$$
\begin{aligned}
& x \mu \otimes\left(x+\omega_{1} x^{2}+\cdots+\omega_{n} x^{n+1}+\cdots\right)=x \mu+x^{2} \omega_{1} \mu^{2}+\cdots \\
& +x^{n+1} \omega_{n} \mu^{n+1}+\cdots \text {, } \\
& \left(x+\omega_{1} x^{2}+\cdots+\omega_{n} x^{n+1}+\cdots\right) \otimes x \mu=x \mu+x^{2} \omega_{1} \mu+\cdots \\
& \text { whence } \\
& +x^{n+1} \omega_{n} \mu+\cdots \text {, }
\end{aligned}
$$

$$
\begin{aligned}
x \mu & \otimes\left(x+\omega_{1} x^{2}+\cdots+\omega_{n} x^{n+1}+\cdots\right) \otimes(x \mu)^{-1} \\
& =x+x^{2} \omega_{1} \mu+\cdots+x^{n+1} \omega_{n} \mu^{n}+\cdots .
\end{aligned}
$$


Since $\left\{S(\mu), \mu \in \Omega^{*}\right\}$ is a subgroup the factor set of $\Omega^{*}$ with respect to $G$ is the unit factor set.

CoROLlaRY 2. The group $G_{1}$ is a complete metric group; if the field $\Omega$ is finite then $G_{1}$ is compact.

Proof. The system of normal subgroups $G_{i}$ defines a topology in $G_{1}$. The group $G_{1}$ is complete with respect to this topology for $G_{1}$ contains all elements $x+\omega_{1} x^{2}+\cdots+\omega_{n} x^{n+1}+\cdots$ with arbitrary coefficients $\omega_{n}$. We next define a metric in $G_{1}$ which is consistent with the system of defining neighborhoods $G_{i}, i=1,2, \ldots$. Suppose $S=x+\alpha_{1} x^{2}+\cdots+\alpha_{n-1} x^{n}+\alpha_{n} x^{n+1}+\cdots, T=x+\beta_{1} x^{2}+\cdots$ $+\beta_{n-1} x^{n}+\beta_{n} x^{n+1}+\cdots$ are two arbitrary elements of $G_{1}$. We define $\delta(S, T)$ to be $e^{-n}$ if and only if $\alpha_{i}=\beta_{i}$ for all $i<n$ and $\alpha_{n} \neq \beta_{n}$. Then $\delta(S, T)=\delta(T, S), \delta(S, T)>0$ for $S \neq T, \delta(S, T) \leqq \max [\delta(S, R)$, $\delta(T, R)]$. Moreover $G_{n}$ consists of all elements $S$ in $G_{1}$ which have distance $\delta(1, S) \leqq e^{-n}$. Thus $G_{1}$ is a 0 -dimensional metric group. The factor groups $G_{1} / G_{n}$ contain nontrivial elements of finite order if and only if $\chi<\infty$ holds for the characteristic of $\Omega$. If $\chi<\infty$ the elements of $G_{1} / G_{n}$ have at most order $\chi^{n-1}$. Hence $\lim _{n \rightarrow \infty} g^{x^{n}}=1$ for each element $g$ of $G_{1}$, thus $G_{1}$ is a generalized $\chi$-adic group. ${ }^{10}$ In particular, $G_{1}$ is compact if $\Omega$ is finite. In this case $G_{n} / G_{n+1} \cong \Omega$, and thus $G_{1}$ is isomorphic to the inverse $\operatorname{limit} \lim _{n \rightarrow \infty} G_{1} / G_{n}$ where the factor groups $G_{1} / G_{n}$ are all finite.

Definition 3. The totality of elements $S$ in $G_{1}$ with $a^{S} \equiv a\left(\bmod \mathfrak{P}^{n}\right)$ for each $a$ in $D$ is called the nth pseudo-ramification group $R_{n}$ of $F_{1}{ }^{11}$

THEOREM 3. The groups $G_{n}$ and $R_{n}$ coincide.

Proof. We first remark that the first pseudo-ramification group $R_{1}$ coincides with $G_{1}$, for the general element $x+\omega_{1} x^{2}+\cdots$ of $G_{1}$ induces the identity mapping on the residue class field $\Omega$ of $F$. Suppose now that $g=x+\sum_{\nu=n+1}^{\infty} \omega_{\nu} x^{\nu}$ is an arbitrary element of $G_{n}$. Let $a=\alpha_{0}+\alpha_{1} t+\cdots+\alpha_{n-1} t^{n-1}+\alpha_{n} t^{n}+\cdots$. Then

$$
\begin{aligned}
a^{0}= & \alpha_{0}+\alpha_{1}\left(t+\sum_{\nu=n}^{\infty} \omega_{\nu} t^{\nu}\right)+\cdots+\alpha_{n-1}\left(t+\sum_{\nu=n}^{\infty} \omega_{\nu} t^{\nu}\right)^{n-1} \\
& +\alpha_{n}\left(t+\sum_{\nu=n}^{\infty} \omega_{\nu} t^{\nu}\right)^{n}+\cdots
\end{aligned}
$$

${ }_{10}$ A topological group $G$ is called $x$-adic, if $\lim _{n \rightarrow \infty} g^{x^{n}}=1$ for each $g$ in $G, x<\infty$, in a sense of the topology of $G$. Examples of $\chi$-adic groups are the additive subgroup of the $\chi$-adic number field and multiplicative groups in $\chi$-adic algebras.

11 See $[6$, p. 438]. 


$$
\begin{aligned}
= & \alpha_{0}+\alpha_{1} t+\alpha_{1} \sum_{\nu=n}^{\infty} \omega_{\nu} t^{\nu}+\cdots+\alpha_{n-1} t^{n-1}+\alpha_{n-1}(n-1) t^{n} \omega_{n} \\
& +\cdots+\alpha_{n} t^{n}+\cdots+\alpha_{n} n t^{n+\nu} \omega_{n}+\cdots \\
\equiv & \alpha_{0}+\alpha_{1} t+\cdots+\alpha_{n-1} t^{n-1}\left(\bmod \mathfrak{P}^{n}\right),
\end{aligned}
$$

whence $G_{n} \subseteq R_{n}$. Conversely, let $g \in R_{n}$ and $t^{0}=t+\beta_{1} t^{2}+\cdots+\beta_{n-1} t^{n}$ $+\beta_{n} t^{n+1}+\cdots$. We compute

$$
\begin{aligned}
& a^{o}=\alpha_{0}+\alpha_{1}\left(t+\beta_{1} t^{2}+\cdots+\beta_{n-1} t^{n}+\beta_{n} t^{n+1}+\cdots\right) \\
& +\alpha_{2}\left(t+\beta_{1} t^{2}+\cdots\right)^{2}+\cdots+\alpha_{n-1}\left(t+\beta_{1} t^{2} \cdots\right)^{n-1}+\cdots \\
& \equiv \alpha_{0}+\alpha_{1} t+\alpha_{1} \beta_{1} t^{2}+\cdots+\alpha_{2} t^{2}+2 \alpha_{2} \beta_{1} t^{3}+\cdots+\cdots \\
& +\alpha_{n-1} t^{n-1}+\cdots \\
& \equiv \alpha_{0}+\alpha_{1} t+\alpha_{2} t^{2}+\cdots+\alpha_{n-1} t^{n-1}\left(\bmod \mathfrak{B}^{n}\right) \text {. }
\end{aligned}
$$

Reducing this congruence successively $\bmod \mathfrak{P}^{2}, \mathfrak{P}^{8}, \cdots, \mathfrak{\beta}^{n-1}$ we ob$\operatorname{tain} \beta_{1}=\cdots=\beta_{n-2}=0$, whence $G_{n} \supseteq R_{n}$.

REMARK. As in the ordinary Hilbert theory it follows that $R_{n}$ is an invariant subgroup of $G_{1}$. This furnishes a new proof for the invariance of the groups $G_{n}$.

THEOREM 4. Suppose that $H$ is a finite subgroup of $G_{1}$ and let $K$ be the associated subfield of $F$. The ramification groups $H_{i}$ of $F / K$ are equal to $G_{i} \cap H$.

PRoof. Let $V_{K}$ be the valuation which is induced by $V$ on the subfield $K$. Suppose that $\Gamma(F)$ and $\Gamma(K)$ are the value groups associated to $V$ and $V_{K}$, respectively. We first observe that $\Omega$ is contained in $K$ for $H \subset G_{1}$. Let $\bar{K}$ be the completion of $K$ with respect to $V_{K}$, then $K \subseteq \bar{K} \subseteq F$ for the fundamental sequences in $K$ are special fundamental sequences in $F$ by the definition of $V_{K}$. Next observe that $V_{K}$ has only $V$ for its prolongation to $F$. Remark that all prolongations of $V_{K}$ are given as $V^{h}, h$ in $H$; thus, by Lemma $1, V^{h}=V$. Combining these facts, we find $[F: K]=[\Gamma(F): \Gamma(K)]=[\Gamma(F): \Gamma(\bar{K})]=[F: \bar{K}]$. Consequently $K=\bar{K}$, that is, $K$ is a complete field and $H$ is the decomposition and inertial group for $F / K$. Now let $H_{i}$ be the $i$ th ramification group of $F / K$. Then $H_{i} \subset R_{i}=G_{i}$ by the proof of Theorem 3 . Thus $H_{i} \subseteq R_{i} \cap H$. Conversely, let $S$ be an element of $R_{i} \cap H$. Then $a^{S} \equiv a\left(\bmod \mathfrak{B}^{i}\right)$ by definition of $R_{i}$. Hence $S$ lies in $H_{i}$. Therefore $H_{i}=R_{i} \cap H$ as asserted.

Let $K$ be an arbitrary subfield of $F$ such that $\Omega \subset K$ and $[F: K]$ $<\infty$. The field $K$ is then complete with respect to the valuation $V_{\boldsymbol{K}}$ 
induced by $V$. For the proof we observe that the valuation $V$ has a unique prolongation to the normal closure of $F$ over $K$, for the latter is complete since it is a finite extension of $F$. The ramification theory implies, as at the beginning of the proof for Theorem 4, that $K$ is complete. Now let $\pi(F)$ be any prime element of $F$, that is $V \pi(F)=1$, $F=\Omega\{\pi(F)\}$. Then $N_{F / K} \pi(F)$ is a prime element $\pi(K)$ for $K$ because $V \pi(K)=[F: K]$ and $\Omega\{\pi(K)\}=K$ by Lemma 2 applied to the chain $\Omega\{\pi(K)\} \subseteq K \subset F$. We have $\pi(K)=\sum_{\nu=0}^{\infty} \omega_{\nu} \pi(F)^{n+\nu}$, where $[F: K]=n$ and $\omega_{0} \neq 0$. Applying an automorphism $S$ of $G$ to $\pi(F)$ we obtain a field $K^{S}=\Omega\left\{\pi(K)^{S}\right\}$ which is isomorphic to $K$ and $K=K^{S}$ if and only if $S$ is an automorphism of $F / K$. The group $S H S^{-1}$ is the full automorphism group of $F / K^{s}$ if $H$ is the automorphism group of $F / K$. We apply these results to prove the following theorem.

THEOREM 5. The automorphism group $G$ of $F$ contains elements of finite order.

Proof. We first observe that $F$ always has normal completely ramified extensions $M$. The existence of such extensions depends on the nature of the residue class field $\Omega^{12}$ In case that $n$ is an integer prime to the characteristic $\chi$ and $\Omega$ contains a primitive $n$th root of unity the field $F$ surely has at least one cyclic completely ramified extension of degree $n$. For example, there is surely such a quadratic extension if $(\chi, 2)=1$ or $\chi=\infty$. If $\chi$ is finite then $\Omega$ contains some field of $\left(\chi^{m}-1\right)$ th roots of unity, $m \geqq 1$, and any $n \mid\left(\chi^{m}-1\right)$ may be used. For finite $\chi$ there are infinitely many cyclic completely ramified extensions of degree $\chi$ over $F .{ }^{13}$ Hence we know that completely ramified normal extensions $M / F$ exist. Since $M$ is completely ramified, say of degree $n$, we have $\pi(F)=\sum_{\nu=0}^{\infty} \omega_{\nu} \pi(M)^{\nu+n}, \omega_{0} \neq 0$, for a prime element $\pi(M)$ of $M$. By the structure theory of fields of power series the fields $M$ and $F$ are isomorphic. ${ }^{14}$ Suppose that $\phi$ is a realization of the isomorphism $M \cong F$, that is, $M^{\phi}=F$. We apply $\phi$ to the equation for $\pi(F)$ and obtain $\pi(F)^{\phi}=\sum_{\nu=0}^{\infty} \omega_{\nu}\left[\pi(M)^{\phi}\right]^{n+\nu}$. Then $\pi(F)^{\phi}$ determines by $\Omega\{\pi(F) \phi\}$ a subfield $K$ of $F$ so that the Galois groups of $M / F$ and $F / \Omega\left\{\pi(F)^{\phi}\right\}$ are isomorphic. Observing that the Galois group of $M / F$ is isomorphic to a finite subgroup of $G$, the assertion of the theorem follows. ${ }^{15}$

As a special case we consider cyclic subgroups $H$ of order $n$ in $G$ for which $(n, \chi)=1$. Let $K$ be the field of invariants for $H$. We have

\footnotetext{
${ }^{12}$.See $[11,14]$.

${ }^{18}$ See [14].

${ }^{14}$ See $[1,6,13,14]$.

${ }^{16}$ See $[3$, p. 890].
} 
$K=\Omega\left\{u t^{n}\right\}$ where $u$ is a unit of $F$. Then $u=\omega v$ where $\omega \neq 0$ lies in $\Omega$ and $v \equiv 1(\bmod P)$. We have $K=\Omega\left\{v t^{n}\right\}$, for $K$ is an immediate extension of $\Omega\left\{v t^{n}\right\}$. Since $(n, \chi)=1$ there exists ${ }^{16}$ a unit $y$ of $F$ with $y \equiv 1(\bmod P), y^{n}=v$. Therefore $K=\Omega\left\{(y t)^{n}\right\}$. Since $\Omega\{y t\}=\Omega\{t\}=F$ we obtain $H=S H_{0} S^{-1}$ where $S$ is the automorphism of $G_{1}$ with $t^{S}=y t$ and $H_{0}$ is the finite subgroup of $G$ generated by the element $\zeta x, \zeta$ a primitive $n$th root of unity. Finally $K=\Omega\left\{t^{n}\right\}$ if and only if $S H_{0} S^{-1}$ $=H_{0}$, that is, $S$ induces an automorphism on $H_{0}$. Expressing $K=\Omega\left\{t^{n}\right\}$ in terms of the prime elements we have $(y t)^{n} / t^{n}=y^{n}$ lies in $\Omega\left\{t^{n}\right\}$. Consequently $y^{n}$ must be a power series $1+\sum_{j=1}^{\infty} \alpha_{j}\left(t^{n}\right)^{j}$. Conversely each unit $y$ with $y^{n}$ in $\Omega\left\{t^{n}\right\}$ gives rise to $K=\Omega\left\{t^{n}\right\}$. Since there are always elements $y$ for which $y^{n}$ does not lie in $\Omega\left\{t^{n}\right\}$ and $y^{n}=1+\omega t$ $+\cdots, \omega \neq 0$, we have the existence of automorphisms $S$ with $S H_{0} S^{-1} \neq H_{0}$ and $\Omega\left\{t^{n}\right\} \cap \Omega\left\{(y t)^{n}\right\}=\Omega$, as can be shown by comparing the series of the fields. This shows that the set of normal subfields $K$ with $[F: K]<\infty,([F: K], \chi)=1$ is not a lattice. We remark that conditions on $y$ may be derived to describe $\Omega\left\{(y t)^{s}\right\}=\Omega\left\{t^{*}\right\}$ for $s \mid n$.

The group $G$ can be interpreted as a group of automorphisms of an infinite Lie ring $\mathfrak{D}_{L}$ over $\Omega$. We define $\mathfrak{D}_{L}$ as the ring $\mathfrak{D}$, considered as an infinite vector space over $\Omega$, in which a product $[f, g]$ is defined as follows. We set $[f, g]=(d g / d t) f-(d f / d t) g$. The rules of differentiation imply that $[f, g]$ obeys the rules of the Jacobi bracket. For a basis of $\mathfrak{D}_{L}$ we may take the elements $e_{i}=t^{i+1}, i=-1,0,1, \cdots$ and $\left[e_{i}, e_{j}\right]=(j-i) e_{i+j}$. By actual computation it can be shown that the mapping

$$
f(t) \rightarrow f\left(t^{S}\right) /\left(d t^{S} / d t\right)
$$

is an automorphism of $\mathfrak{D}_{L} \cdot{ }^{17}$ Moreover, distinct elements of $G$ give rise to distinct automorphisms in the automorphism group $\boldsymbol{A}\left(\mathfrak{D}_{L}\right)$ of $\mathfrak{D}_{L}$.

TheOREM 6. The groups $G$ and $A\left(\mathfrak{D}_{L}\right)$ coincide if $\chi=\infty$.

Proof. Suppose that $\Sigma$ is an automorphism of $\mathcal{D}_{L}$. Let $t^{\Sigma}=\Phi(t)$. We shall show that there exists an automorphism $S$ in $G$ so that $\Sigma$ is determined by the formula $\left({ }^{* *}\right)$ applied to $t$, that is, $t^{\Sigma}=t^{s} /\left(d t^{s} / d t\right)$. Such an element $S$ determines then the automorphism $\Sigma$ on all of $\mathfrak{D}_{L}$ because the elements $t^{i+1}$ form a basis of $\mathfrak{D}_{L}$. To determine $S$ it suffices to find a unique element $\phi(t)=\alpha_{1} t+\alpha_{2} t^{2}+\cdots, \alpha_{1} \neq 0$, for which $t^{\Sigma}=\Phi(t)=f[\phi(t)] /(d \phi / d t)$. By formal integration we find

\footnotetext{
${ }^{10}$ See $[10$, p. $561 ; 11$, p. 441$]$.
}

${ }_{17}$ Compare [15, pp. 37-47]. 
$\phi(t)=\exp \left[\int \Phi^{-1}(t) d t\right]$ where $\int \Phi^{-1}(t) d t$ is the formal indefinite integral without a constant of integration. We observe that all formal operations involved can be carried out because they are determined in $\mathfrak{D}$. The automorphism $S$ is given by $\phi(x)$ using the representation of $G$ by $U$. In concluding we remark that $G \subset A\left(\mathfrak{D}_{L}\right)$ for $\chi<\infty$. The inequality may be explained by the fact that $t^{n}$ is never the derivative of an element in $F$ if $n \equiv 0(\bmod \chi)$.

\section{BIBLIOGRAPHY}

1. H. Hasse and F. K. Schmidt, Die Struktur diskret bewerteter Körper, J. Reine Angew. Math. vol. 170 (1934) pp. 4-63.

2. H. Hasse, Noch eine Begründung der Theorie der höheren Differentialquotienten in einem algebraischen Funktionenkörper einer Unbestimmten, J. Reine Angew. Math. vol. 177 (1937) pp. 215-237. 894.

3. N. Jacobson, A note on topological fields, Amer. J. Math. vol. 59 (1937) pp. 889-

4. W. Krull, Allgemeine Bervertungstheorie, J. Reine Angew. Math. vol. 167 (1932) pp. $160-196$.

5. Saunders MacLane, The uniqueness of the power series representation of certain fields with valuations, Ann. of Math. vol. 39 (1938) pp. 370-382.

6. - Subfields and automorphism groups of p-adic fields, Ann. of Math. vol. 40 (1939) pp. 423-442.

7. Saunders MacLane and O. F. G. Schilling, Normal algebraic number fields, Trans. Amer. Math. Soc. vol. 50 (1941) pp. 295-384.

8. O. Ore, Linear equations in non-commutative fields, Ann. of Math. vol. 32 (1931) pp. $463-477$.

9. - Theory of non-commutative polynomials, Ann. of Math. vol. 34 (1933) pp. $480-508$.

10. O. F. G. Schilling, Arithmetic in fields of formal power series in several variables, Ann. of Math. vol. 38 (1937) pp. 551-576.

11. - Regular normal extensions over complete fields, Trans. Amer. Math. Soc. vol. 47 (1940) pp. 440-454.

12. F. K. Schmidt, Mehrfach perfekte Körper, Math. Ann. vol. 108 (1933) pp. 1-25.

13. O. Teichmüller, Diskret bewertete perfekte Körper mit unvollkommenem Restklassenkörper, J. Reine Angew. Math. vol. 176 (1936) pp. 141-152.

14. E. Witt, Zyklische Körper und Algebren der Charakteristik p vom Grade $p^{n}$, J. Reine Angew. Math. vol. 176 (1936) pp. 126-140.

15. H. Zassenhaus, Über Lie'sche Ringe mit Primzahlcharakteristik, Abh. Math. Sem. Hansischen Univ. vol. 13 (1939) pp. 1-100.

The University of Chicago 